\title{
Influence of Vacancy Defect on Surface Feature and Adsorption of Cs on GaN(0001) Surface
}

\author{
Yanjun Ji, ${ }^{1}$ Yujie Du, ${ }^{1,2}$ and Meishan Wang ${ }^{3}$ \\ ${ }^{1}$ Department of Optoelectronic Engineering, Binzhou University, Binzhou 256603, China \\ ${ }^{2}$ Institute of Electronic Engineering and Opto-Electric Technology, Nanjing University of Science and Technology, \\ Nanjing 210094, China \\ ${ }^{3}$ School of Physics and Optoelectronic Engineering, Ludong University, Yantai 264025, China \\ Correspondence should be addressed to Yujie Du; duyujie442@163.com
}

Received 21 March 2014; Accepted 13 May 2014; Published 7 July 2014

Academic Editor: Fouad El Haj Hassan

Copyright (C) 2014 Yanjun Ji et al. This is an open access article distributed under the Creative Commons Attribution License, which permits unrestricted use, distribution, and reproduction in any medium, provided the original work is properly cited.

The effects of $\mathrm{Ga}$ and $\mathrm{N}$ vacancy defect on the change in surface feature, work function, and characteristic of Cs adsorption on a $(2 \times 2) \mathrm{GaN}(0001)$ surface have been investigated using density functional theory with a plane-wave ultrasoft pseudopotential method based on first-principles calculations. The covalent bonds gain strength for Ga vacancy defect, whereas they grow weak for $\mathrm{N}$ vacancy defect. The lower work function is achieved for $\mathrm{Ga}$ and $\mathrm{N}$ vacancy defect surfaces than intact surface. The most stable position of Cs adatom on Ga vacancy defect surface is at $\mathrm{T}_{1}$ site, whereas it is at $\mathrm{B}_{\mathrm{Ga}}$ site on $\mathrm{N}$ vacancy defect surface. The $E_{\text {ads }}$ of Cs on $\mathrm{GaN}(0001)$ vacancy defect surface increases compared with that of intact surface; this illustrates that the adsorption of Cs on intact surface is more stable.

\section{Introduction}

Due to its important characteristics such as wide band gap, high thermal conductivity, high breakdown voltage, high melting point, and chemical stability, among others, $\mathrm{GaN}$ and its compounds such as $\mathrm{GaN}_{x} \mathrm{As}_{1-x}$ [1-3] have emerged as a new type material for the fabrication of optoelectronic devices in the blue and ultraviolet spectral region [4-10]. Most research has focused on its physical properties, growth mechanisms, and surface structures [1115]. The (0001) surface of Wurtzite GaN has good surface state and simple production process [16], the surface size of $\mathrm{GaN}(0001)$ is different, and the reconstruction is different in atomic structure $[17,18]$. The $2 \times 2$ surface is considered the most stable surface [19], while a $2 \times 2 \mathrm{Ga}$ adatom model is thermodynamically favored under moderately Garich conditions [20]. The surface stability and conductivity properties of $\mathrm{GaN}(0001)$ are superior to that of $(000 \overline{1})[13$, 21]. GaN(0001) surface becomes the focus of attention of optoelectronics community. The effective negative electron affinity has been demonstrated for $p$-GaN(0001) surfaces after Cs adsorption or Cs and O activating [22-27]; this is important for vacuum-type optoelectronic devices.

Defects may be produced in GaN materials growth process and interact with carriers. The existence of defects will affect the adsorption of Cs on GaN surface and then affect the performances of photoelectric devices. Theoretical studies of the eigen defects of GaN have been reported [2830]; however, the influence of such defects on the adsorption of $\mathrm{Cs}$ on $\mathrm{GaN}$ surface has not been determined. In this paper, we employ plane-wave with ultrasoft pseudopotential method to study the influence of $\mathrm{Ga}$ and $\mathrm{N}$ vacancy defects on the surface feature and adsorption of $\mathrm{Cs}$ on $\mathrm{GaN}(0001)$ surface based on the first-principle density functional theory (DFT).

\section{Computational Methods}

The parameters for the optimized bulk wurtzite GaN structures are $a=b=0.3189 \mathrm{~nm}$ and $c=0.5185 \mathrm{~nm}$ [31]. All calculations were performed with the quantum mechanics 
program Cambridge Serial Total Energy Package [32] based on density functional theory (DFT). The Broyden-FletcherGoldfarb-Shanno algorithm was used to relax the structure of the crystal model. The convergence precision was set to an energy change $<2 \times 10^{-6} \mathrm{eV} /$ atom, force $<0.005 \mathrm{eV} / \mathrm{nm}$, convergence tolerance of a single atomic energy $<1 \times$ $10^{-5} \mathrm{eV} /$ atom, stress $<0.05 \mathrm{GPa}$, and change in displacement $<0.0001 \mathrm{~nm}$ in an iterative process. The surface slab was modeled with six $\mathrm{GaN}(0001)$ bilayers of which the lower three bilayers were fixed in the bulk configurations and a vacuum region equivalent to six $\mathrm{GaN}$ bilayers with overall approximate length of $1.3 \mathrm{~nm}$ was required. The bottom side of the slab was saturated with pseudohydrogen atoms to prevent transfer of surface charges (shown in Figure 1). Wave functions were expanded in a plane-wave basis set up to an energy cutoff of $400 \mathrm{eV}$ and integrations over the Brillouin zone were performed using a $4 \times 4 \times 1$ Monkhorst-Pack set sampling-point scheme for the surface supercell. The electron exchange and correlation were treated by using the PerdewBurke-Ernzerhof (PBE) formulation of the generalized gradient approximation (GGA) [33].

One $\mathrm{Ga}$ atom or one $\mathrm{N}$ atom on the outmost layer of $2 \times 2 \mathrm{GaN}(0001)$ surface was removed in researching vacancy defect surface, respectively (shown in Figure 1). Figure 1(a) shows the side view of Ga vacancy defect surface and (c) is the top view. Figure 1(b) shows the side view of $\mathrm{N}$ vacancy defect surface and (d) is the top view. For a Cs adsorption on $\mathrm{GaN}(0001)$ surface, five typical adsorption models including sites of $\mathrm{T}_{1}$ (Ga top), $\mathrm{H}_{3}$ (hollow site), $\mathrm{T}_{4}$ ( $\mathrm{N}$ top), $\mathrm{B}_{\mathrm{Ga}}$ (Ga bridge), and $\mathrm{B}_{\mathrm{N}}$ (N bridge) were chosen $[34,35]$. In this paper, these five typical adsorption sites are adopted to study the influence of $\mathrm{Ga}$ and $\mathrm{N}$ vacancy defects on the adsorption of $\mathrm{Cs}$ on $\mathrm{GaN}(0001)$ surface. Figure 2 shows the top view of $\mathrm{Cs}$ at $\mathrm{T}_{1}, \mathrm{H}_{3}, \mathrm{~T}_{4}, \mathrm{~B}_{\mathrm{Ga}}$, and $\mathrm{B}_{\mathrm{N}}$ sites on $\mathrm{GaN}(0001)$ defect surface. Figure 2(a) shows the top view of Cs on Ga vacancy defect surface and Figure 2(b) is Cs on N vacancy defect surface.

\section{Deficient Surface}

Based on the mean of atoms coordinates, the thickness of the first bilayer is calculated; it is $0.0272 \mathrm{~nm}$ for Ga vacancy defect surface, whereas it is $0.0436 \mathrm{~nm}$ for $\mathrm{N}$ vacancy defect surface and $0.0653 \mathrm{~nm}$ for intact surface, compared with an ideal value $0.0647 \mathrm{~nm}$. It has the biggest change in surface feature for Ga vacancy defect surface after relaxation. To study the reason for the change of surface feature, the charges of atoms in the outmost layer are shown in Table 1; the overlap populations and bond lengths between $\mathrm{Ga}$ and $\mathrm{N}$ atoms in the outmost layer of intact, Ga vacancy defect, and $\mathrm{N}$ vacancy defect surfaces are shown in Table 2.

The overlap population increases, the bond length decreases, and the covalent bond between $\mathrm{Ga}$ and $\mathrm{N}$ gains strength for Ga vacancy defect surface as shown in Table 2 caused mainly by the increase of charges of every Ga atom (see Table 1) compared with that of intact surface.

The overlap population decreases, covalent bond is weakened, and bond length between $\mathrm{Ga}$ and $\mathrm{N}$ increases for $\mathrm{N}$ vacancy defect surface as shown in Table 2 caused mainly by the decrease of charges of $\mathrm{N}$ and Ga atoms (see Table 1) compared with that of intact surface.

The main reason for the change of surface feature is the break of atoms bonds in the outmost surface layer, there are uncompensated electrons, and dipole moment directing to outside is formed. Compared with the intact surface, the charges of every $\mathrm{Ga}$ atom in $\mathrm{Ga}$ vacancy defect surface increases, while the total charges of $\mathrm{Ga}$ atoms in the outmost layer decrease, so the dipole moment increases. The thickness of the first bilayer is compacted by means of strong dipole moment and covalent bond. There is a preponderance of uncompensated electrons for $\mathrm{N}$ vacancy defect surface; the dipole moment is the max, but the change in the first layer thickness is less than that of Ga vacancy defect surface by means of the weak covalent bond.

For semiconductors, the work function is the minimum energy needed by electrons at the bottom of the semiconductor to escape externally. The easier the escape is, the larger dipole moment directing to outside is formed, the lower work function is. The size of the dipole moment among three surfaces is intact surface $<\mathrm{Ga}$ vacancy defect surface $<\mathrm{N}$ vacancy defect surface. Work functions of three surfaces are $4.20 \mathrm{eV}$ (in agreement with Re.18), $4.054 \mathrm{eV}$, and $4.052 \mathrm{eV}$, respectively (shown in Table 3 ). Calculations show that the defect favors the escape of electrons but can induce bigger change in surface feature.

The charge density difference in the (0001) plane of the surface atoms is shown in Figure 3 to indicate the influence of $\mathrm{Ga}, \mathrm{N}$ defect on the interaction between atoms visually, in which the bond length is also given. The electron cloud of the $\mathrm{N}$ atoms is uniformly distributed in three directions, joining to $\mathrm{Ga}$ atoms in the (0001) plane for intact surface, as shown in Figure 3(a). The degree of electron aggregation enlarges after Ga defect (Figure 3(b)), the overlap population increases, the covalent bond of $\mathrm{Ga}-\mathrm{N}$ is stronger and the bond length of Ga-N is shorter than that of intact surface. The overlap population between the $\mathrm{Ga}$ and $\mathrm{N}$ atoms gets its minimum after $\mathrm{N}$ defect (Figure 3(b)); the bond length of Ga$\mathrm{N}$ increases.

\section{Cs Adsorption on $\mathrm{GaN}(0001)$ Deficient Surface}

To study the effect of defect on the adsorption of Cs on $\mathrm{GaN}(0001)$ surface, first, a $\mathrm{Cs}$ was placed at $\mathrm{H}_{3}$ site on $\mathrm{Ga}$ and $\mathrm{N}$ vacancy defect surfaces above surface $0.1 \mathrm{~nm}$ moving freely in relaxation process and, then, was fixed at five high symmetry sites moving only in $Z$ direction.

Adsorption energy $E_{\text {ads }}$ was calculated as the difference between the total energy of the $\mathrm{GaN}(0001)$ slab with adsorbed Cs and the sum of the total energies of the clean surface and isolated Cs atom [25]. The $E_{\text {ads }}$ of a Cs at these six sites on $\mathrm{Ga}$ vacancy defect surface is shown in Table 3. The results show that $E_{\text {ads }}$ is negative no matter Cs is at the five high symmetry sites or moves freely; it means that the absorption process is an exothermic chemical process and is stable. The final convergence position of $\mathrm{Cs}$ is above the Ga vacancy when it moves freely on Ga vacancy defect surface. The $E_{\text {ads }}$ of every 


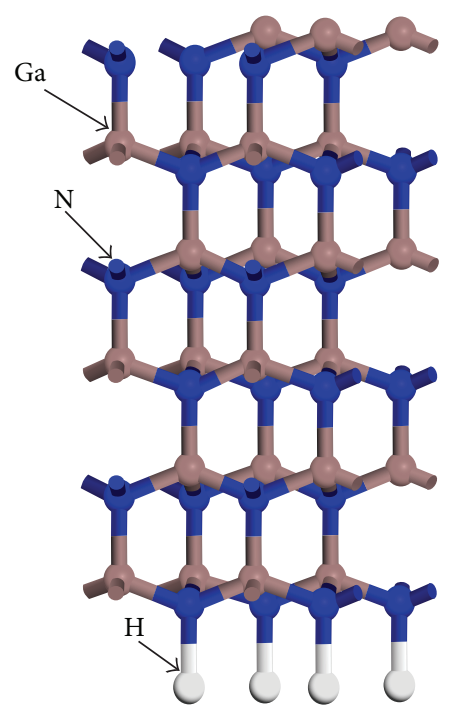

(a)

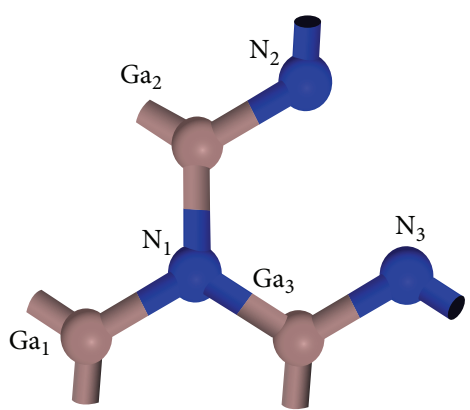

(c)

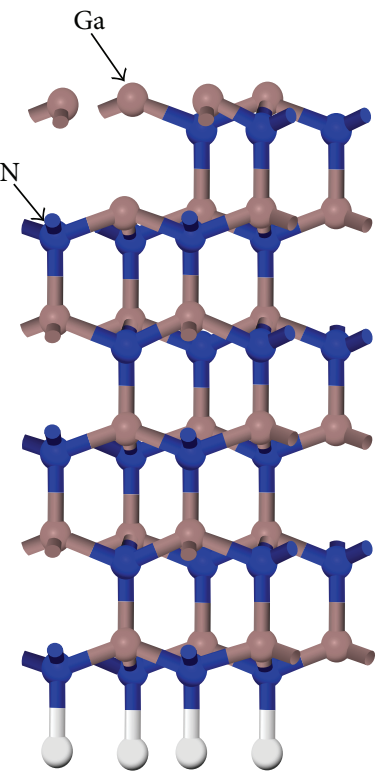

(b)
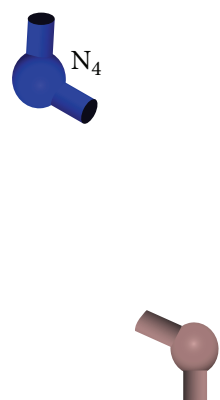

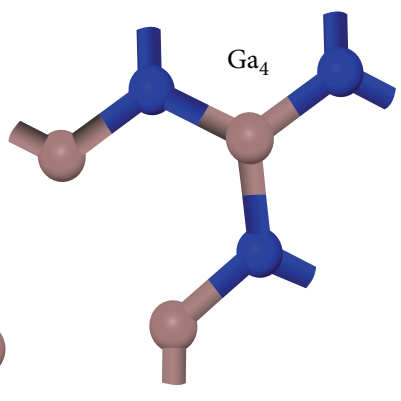

(d)

Figure 1: $2 \times 2 \mathrm{GaN}(0001)$ surface: (a) side view of Ga vacancy defect surface, (b) side view of N vacancy defect surface, (c) top view of Ga vacancy defect surface, and (d) top view of $\mathrm{N}$ vacancy defect surface. $\mathrm{Ga}$ and $\mathrm{N}$ atoms are marked.

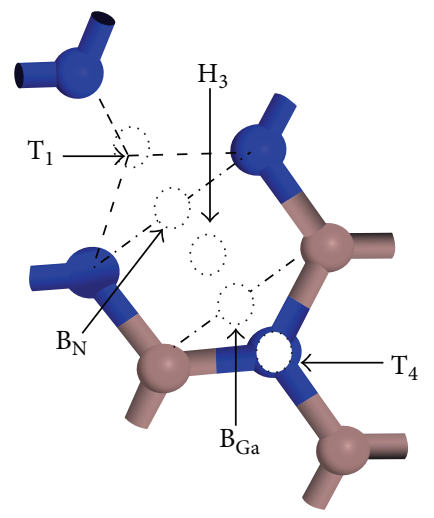

(a)

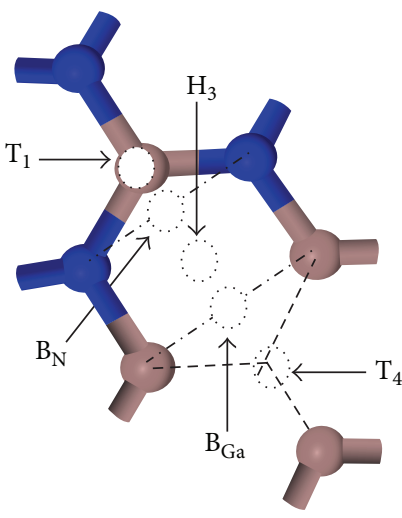

(b)

Figure 2: Top view of various possible adsorption sites of Cs on $2 \times 2 \mathrm{GaN}(0001)$ defect surface: (a) Cs on Ga defect surface, (b) Cs on N defect surface. 
TABLE 1: Charges of atoms in the outmost layer of intact, Ga vacancy defect, and N vacancy defect surfaces.

\begin{tabular}{lccccccrcr}
\hline Surface & \multicolumn{5}{c}{ Atoms and its charges/e } & $\mathrm{N}_{1}$ & $\mathrm{~N}_{2}$ & $\mathrm{~N}_{3}$ \\
\hline Intact & $\mathrm{Ga}_{1}$ & $\mathrm{Ga}_{2}$ & $\mathrm{Ga}_{3}$ & $\mathrm{Ga}_{4}$ & -0.98 & -0.98 & -0.98 \\
Ga vacancy defect & 0.75 & 0.75 & 0.75 & 0.75 & -0.98 & -0.98 & -0.98 \\
N vacancy defect & & 0.88 & 0.88 & 0.88 & -1.04 & -0.97 \\
\hline
\end{tabular}

TABLE 2: N-Ga bond lengths and overlap populations of intact, Ga vacancy defect, and N vacancy defect surfaces.

\begin{tabular}{|c|c|c|c|c|c|c|c|}
\hline \multirow{2}{*}{ Bonds } & \multicolumn{2}{|c|}{ Intact surface } & \multicolumn{2}{|c|}{ Ga vacancy defect surface } & \multicolumn{3}{|c|}{$\mathrm{N}$ vacancy defect surface } \\
\hline & Overlap population & Bond length/nm & Overlap population & Bond length/nm & Bonds & Overlap population & Bond length/nm \\
\hline $\mathrm{N}_{1}-\mathrm{Ga}_{1}$ & 0.56 & 0.1974 & 0.73 & 0.1868 & $\mathrm{~N}_{2}-\mathrm{Ga}_{4}$ & 0.47 & 0.2053 \\
\hline $\mathrm{N}_{1}-\mathrm{Ga}_{2}$ & 0.55 & 0.1972 & 0.73 & 0.1868 & $\mathrm{~N}_{3}-\mathrm{Ga}_{4}$ & 0.47 & 0.2053 \\
\hline $\mathrm{N}_{1}-\mathrm{Ga}_{3}$ & 0.58 & 0.1967 & 0.65 & 0.1970 & $\mathrm{~N}_{4}-\mathrm{Ga}_{4}$ & 0.53 & 0.2012 \\
\hline
\end{tabular}

TABLE 3: $E_{\text {ads }}$ and work functions of Cs on GaN(0001) intact, Ga vacancy defect, and N vacancy defect surfaces.

\begin{tabular}{lcccccc}
\hline \multirow{2}{*}{ Adsorption site } & \multicolumn{2}{c}{ Intact surface } & \multicolumn{2}{c}{ Ga vacancy defect } & \multicolumn{2}{c}{ N vacancy defect } \\
& $E_{\text {ads }} / \mathrm{eV}$ & Work function/eV & $E_{\mathrm{ads}} / \mathrm{eV}$ & Work function/eV & $E_{\mathrm{ads}} / \mathrm{eV}$ & Work function/eV \\
\hline $\mathrm{H}_{3}$ & -2.02 & 2.16 & -1.15 & 2.46 & 2.28 & -1.55 \\
$\mathrm{~T}_{1}$ & -1.89 & 2.30 & -1.89 & 2.28 & -1.48 & 2.30 \\
$\mathrm{~T}_{4}$ & -1.96 & 2.37 & -0.80 & 2.22 & -1.57 & 2.23 \\
$\mathrm{~B}_{\mathrm{Ga}}$ & -1.98 & 2.36 & -0.84 & 2.33 & -1.54 & 2.20 \\
$\mathrm{~B}_{\mathrm{N}}$ & -2.04 & 2.36 & -0.84 & 2.67 & -1.53 & 2.12 \\
Freedom & -2.04 & 2.42 & -1.99 & & 2.19 \\
\hline
\end{tabular}

symmetry site decreases compared with that of Cs on intact surface. The most stable adsorption site is $\mathrm{T}_{1}$ site (top of $\mathrm{Ga}$ vacancy), no longer the $\mathrm{B}_{\mathrm{N}}$ and $\mathrm{H}_{3}$ sites for intact surface [26]; the most unstable adsorption site is $\mathrm{T}_{4}$ site. The lack of $\mathrm{Ga}$ atom makes the repulsive interaction of $\mathrm{Ga}$ to $\mathrm{Cs}$ nonexistent and Cs be attracted only by three $\mathrm{N}$ atoms, so the stable adsorption site changes compared with that of intact surface. Since the increase of uncomplexed electrons after Ga absence leads to the increasing of dipole moment; work functions of Ga vacancy defect surface with Cs at different high symmetry sites decline slightly compared with that of intact surface.

The $E_{\mathrm{ads}}$ is negative no matter a $\mathrm{Cs}$ is at the five high symmetry sites or moves freely on $\mathrm{N}$ vacancy defect surface (shown in Table 3); it means that the absorption process is also stable. The results show that $E_{\text {ads }}$ of every symmetry site decreases compared with that of Cs on intact surface, while it is bigger than that of Cs on Ga vacancy defect surface. $\mathrm{B}_{\mathrm{Ga}}$ is the most stable adsorption site in agreement with the case when $\mathrm{Cs}$ moves freely on $\mathrm{N}$ vacancy defect surface; $\mathrm{H}_{3}$ is not convergence different from the case of intact surface. Since the increase of uncomplexed electrons after $\mathrm{N}$ absence leads to the increasing of dipole moment, work functions of $\mathrm{N}$ vacancy defect surface in different high symmetry sites decline slightly compared with that of intact surface.
Comparing the $E_{\text {ads }}$ of $\mathrm{Cs}$ on $\mathrm{Ga}$ and $\mathrm{N}$ vacancy defect surfaces, it can be seen that the adsorption of Cs on $\mathrm{N}$ vacancy defect surface is more stable than on Ga vacancy defect surface. The lower work function is achieved for $\mathrm{Ga}$ and $\mathrm{N}$ vacancy defects surfaces than intact surface in agreement with the analysis of dipole moments before.

\section{Discussion and Conclusions}

The change of surface feature has been compared between $\mathrm{GaN}(0001)$ vacancy defect and intact surfaces. Adsorption characteristic and change in work function of a Cs atom on $\mathrm{GaN}(0001)(2 \times 2)$ vacancy defect surface have been investigated using DFT with a plane-wave ultrasoft pseudopotential method based on first-principles calculations, compared with that of intact surface. Results show that $\mathrm{Ga}$ and $\mathrm{N}$ vacancy defects may cause contraction of the first bilayer and decrease of surface work function for the increase of dipole moment. The most stable adsorption site is $\mathrm{T}_{1}$ site (top of Ga vacancy) for $\mathrm{Ga}$ vacancy defect surface, while it is $\mathrm{B}_{\mathrm{Ga}}$ site for $\mathrm{N}$ vacancy defect surface. When the Cs was adsorbed on the vacancy defect surfaces, work functions for different high symmetry sites decline slightly compared with that of intact surface, but $E_{\text {ads }}$ increases, this illustrates that the adsorption of Cs on intact surface is more stable. 


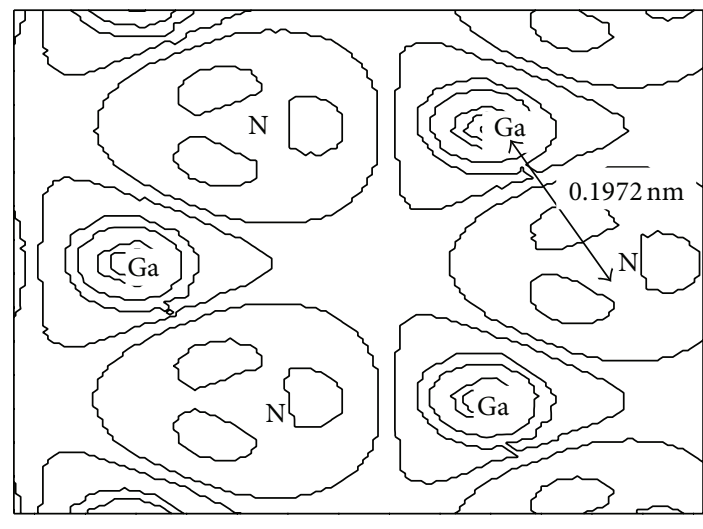

(a)

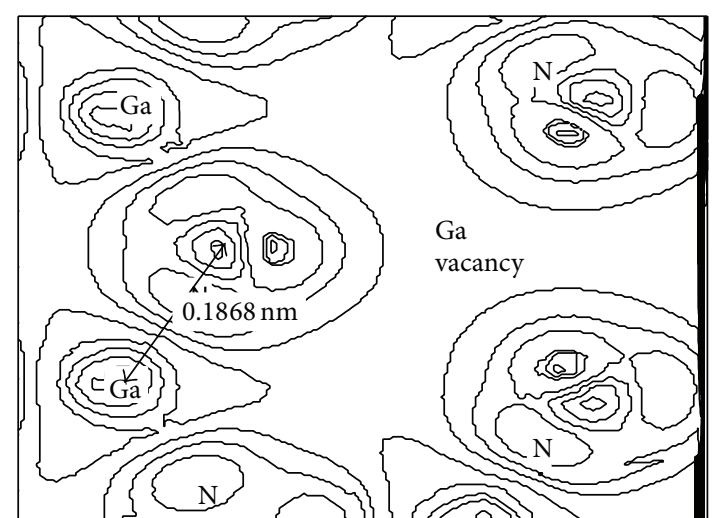

(b)

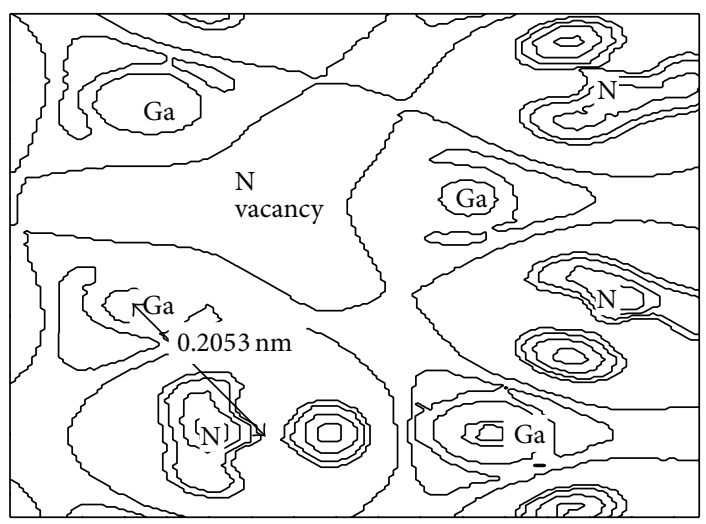

(c)

Figure 3: Electron density difference in the (0001) plane of the surface atoms: (a) intact surface, (b) Ga vacancy defect surface, and (c) N vacancy defect surface.

\section{Conflict of Interests}

The authors declare that there is no conflict of interests regarding the publication of this paper.

\section{Acknowledgments}

The authors acknowledge supports from the National Natural Science Foundation of China (Grant no. 61171042), the Postdoctoral Research Assistance program of Jiangsu (Grant no. 1301008B), the Shandong Natural Science Foundation (Grant no. ZR2013FM003), the Shandong University Science and Technology Project (J14LJ52) and the Binzhou Science and Technology Development Planning (Grant no. 2013ZC0107).

\section{References}

[1] A. H. Reshak, "Dispersion of the second harmonic generation in $\mathrm{GaN}_{x} \mathrm{As}_{1-x}(x=0.25,0.5,0.75)$ alloys," Journal of Alloys and Compounds, vol. 589, pp. 213-217, 2014.

[2] A. H. Reshak, Z. Charifi, and H. Baaziz, "The influence of the lattice relaxation on the optical properties of $\mathrm{GaN}_{x} \mathrm{As}_{1-x}$ alloys," Solar Energy, vol. 90, pp. 134-143, 2013.

[3] H. Baaziz, Z. Charifi, A. H. Reshak, B. Hamad, and Y. Al-Douri, "Structural and electronic properties of $\mathrm{GaN}_{x} \mathrm{As}_{1-x}$ alloys," Applied Physics A, vol. 106, no. 3, pp. 687-696, 2012.
[4] A. Hass Bar-Ilan, S. Zamir, O. Katz, B. Meyler, and J. Salzman, "GaN layer growth optimization for high power devices," Materials Science and Engineering A, vol. 302, no. 1, pp. 14-17, 2001.

[5] H. Zhao, G. Liu, and N. Tansu, "Analysis of InGaN-deltaInN quantum wells for light-emitting diodes," Applied Physics Letters, vol. 97, no. 13, Article ID 131114, 2010.

[6] W. W. Chow, "Modeling excitation-dependent bandstructure effects on InGaN light-emitting diode efficiency," Optics Express, vol. 19, no. 22, pp. 21818-21831, 2011.

[7] R. M. Farrell, P. S. Hsu, D. A. Haeger et al., "Low-thresholdcurrent-density AlGaN-cladding-free $m$-plane InGaN/GaN laser diodes," Applied Physics Letters, vol. 96, no. 23, Article ID 231113, 2010.

[8] J. Zhang, H.-P. Zhao, and N. Tansu, "Large optical gain AlGaNdelta-GaN quantum wells laser active regions in mid- and deepultraviolet spectral regimes," Applied Physics Letters, vol. 98, no. 17, Article ID 171111, 2011.

[9] X. H. Wang, B. K. Chang, Y. J. Du, and J. L. Qiao, "Quantum efficiency of GaN photocathode under different illumination," Applied Physics Letters, vol. 99, no. 4, Article ID 042102, 2011.

[10] X. H. Wang, B. K. Chang, L. Ren, and P. Gao, "Influence of the $p$-type doping concentration on reflection-mode GaN photocathode," Applied Physics Letters, vol. 98, no. 8, Article ID 082109, 2011. 
[11] D. Kapolnek, S. Keller, R. Vetury et al., "Anisotropic epitaxial lateral growth in GaN selective area epitaxy," Applied Physics Letters, vol. 71, no. 9, pp. 1204-1206, 1997.

[12] M. S. Brandt, P. Herbst, H. Angerer, O. Ambacher, and M. Stutzmann, "Thermopower investigation of $n$ - and $p$-type GaN," Physical Review B, vol. 58, no. 12, pp. 7786-7791, 1998.

[13] K. Motohashi, K. Hosoya, M. Imano, S. Tsurubuchi, and A. Koukitu, "Analyses of GaN ( $\left.\begin{array}{llll}0 & 0 & 0 & 1\end{array}\right)$ and $\left(\begin{array}{lll}000 \overline{1}\end{array}\right)$ surfaces by highly-charged ions," Surface Science, vol. 601, no. 22, pp. 53045308, 2007.

[14] Y. Du, B. Chang, X. Fu, X. Wang, and M. Wang, "Electronic structure and optical properties of zinc-blende GaN," Optik, vol. 123, no. 24, pp. 2208-2212, 2012.

[15] V. Timon, S. Brand, S. J. Clark, M. C. Gibson, and R. A. Abram, "First-principles calculations of $2 \times 2$ reconstructions of $\mathrm{GaN}(0001)$ surfaces involving $\mathrm{N}, \mathrm{Al}, \mathrm{Ga}$, In, and As atoms," Physical Review B: Condensed Matter and Materials Physics, vol. 72, no. 3, Article ID 035327, 2005.

[16] S. Nakamura and G. Fasol, The Blue Laser Diode, Springer, Berlin, Germany, 1997.

[17] A. L. Rosa and J. Neugebauer, "First-principles calculations of the structural and electronic properties of clean GaN (0001) surfaces," Physical Review B. Condensed Matter and Materials Physics, vol. 73, no. 20, Article ID 205346, 2006.

[18] A. N. Hattori, K. Endo, K. Hattori, and H. Daimon, "Surface treatments toward obtaining clean $\mathrm{GaN}\left(\begin{array}{llll}0 & 0 & 0 & 1\end{array}\right)$ from commercial hydride vapor phase epitaxy and metal-organic chemical vapor deposition substrates in ultrahigh vacuum," Applied Surface Science, vol. 256, no. 14, pp. 4745-4756, 2010.

[19] K. Rapcewicz, M. B. Nardelli, and J. Bernholc, "Theory of surface morphology of wurtzite GaN (0001) surfaces," Physical Review B. Condensed Matter and Materials Physics, vol. 56, no. 20, Article ID R12725, 1997.

[20] R. M. Feenstra, J. E. Northrup, and J. Neugebauer, "Review of structure of bare and adsorbate-covered GaN(0001) surfaces," MRS Internet Journal of Nitride Semiconductor Research, vol. 7, pp. 1-27, 2002.

[21] Y. Du, B. Chang, J. Zhang, B. Li, and X. Wang, "First-principles study of the electronic structure and optical properties of GaN(0001) surface," Acta Physica Sinica, vol. 61, no. 6, Article ID 067101, 2012 (Chinese).

[22] A. A. Pakhnevich, V. V. Bakin, A. V. Yaz'kov et al., "Energy distributions of photoelectrons emitted from p-GaN(Cs, O) with effective negative electron affinity," JETP Letters, vol. 79, no. 10, pp. 479-483, 2004.

[23] F. Machuca, Z. Liu, J. R. Maldonado, S. T. Coyle, P. Pianetta, and R. F. W. Pease, "Negative electron affinity group IIInitride photocathode demonstrated as a high performance electron source," Journal of Vacuum Science and Technology B: Microelectronics and Nanometer Structures, vol. 22, no. 6, pp. 3565-3569, 2004.

[24] J. Qiao, S. Tian, B. K. Chang, X. Du, and P. Gao, "Progress in study of negative electron affinity GaN vacuum surface electron source," Chinese Physical Society, vol. 58, article 5847, 2009 (Chinese).

[25] X. Du, B. Chang, Y. Qian, R. Fu, P. Gao, and J. Qiao, "Activation technique of GaN negative electron affinity photocathode," Chinese Journal of Lasers, vol. 37, no. 2, pp. 385-388, 2010.

[26] Y. Du, B. Chang, X. Wang, J. Zhang, B. Li, and M. Wang, "Theoretical study of Cs adsorption on GaN( $\left.\begin{array}{llll}0 & 0 & 0 & 1\end{array}\right)$ surface," Applied Surface Science, vol. 258, no. 19, pp. 7425-7429, 2012.
[27] Y. Shen and J. Kang, "Ab initio calculation of the electronic structure of carbon and oxygen impurities in GaN," Acta Physica Sinica, vol. 51, no. 3, article 645, 2002 (Chinese).

[28] C. Pang, J. Shi, Y. Zhang et al., "Electronic structures of wurtzite GaN with Ga and N vacancies," Chinese Physics Letters, vol. 24, no. 7, pp. 2048-2051, 2007.

[29] W. W. Jie and C. Yang, "The electronic structures of vacancy defects in hexagonal GaN," Journal of Sichuan Normal University (Natural Science), vol. 33, article 803, 2010 (Chinese).

[30] Y. Du, B. Chang, H. Wang, J. Zhang, and M. Wang, "First principle study of the influence of vacancy defects on optical properties of GaN," Chinese Optics Letters, vol. 10, no. 5, Article ID 051601, 2012.

[31] P. Perlin, C. Jauberthie-Carillon, J. P. Itie, A. San Miguel, I. Grzegory, and A. Polian, "Raman scattering and x-ray-absorption spectroscopy in gallium nitride under high pressure," Physical Review B, vol. 45, no. 1, pp. 83-89, 1992.

[32] M. D. Segall, P. J. D. Lindan, M. J. Probert et al., "First-principles simulation: ideas, illustrations and the CASTEP code," Journal of Physics Condensed Matter, vol. 14, no. 11, pp. 2717-2744, 2002.

[33] J. P. Perdew, K. Burke, and M. Ernzerhof, "Generalized gradient approximation made simple," Physical Review Letters, vol. 77, no. 18, pp. 3865-3868, 1996.

[34] C. Hu, Y. Chen, J. Li, and Y. Zhang, "First-principles calculations of ethanethiol adsorption and decomposition on GaN ( $\left.\begin{array}{llll}0 & 0 & 0 & 1\end{array}\right)$ surface," Applied Surface Science, vol. 254, no. 20, pp. 6514-6520, 2008.

[35] Q. Sun, A. Selloni, T. H. Myers, and W. A. Doolittle, "Oxygen adsorption and incorporation at irradiated $\mathrm{GaN}(0001)$ and $\mathrm{GaN}(0001)$ surfaces: first-principles density-functional calculations," Physical Review B. Condensed Matter and Materials Physics, vol. 74, no. 19, Article ID 195317, 2006. 

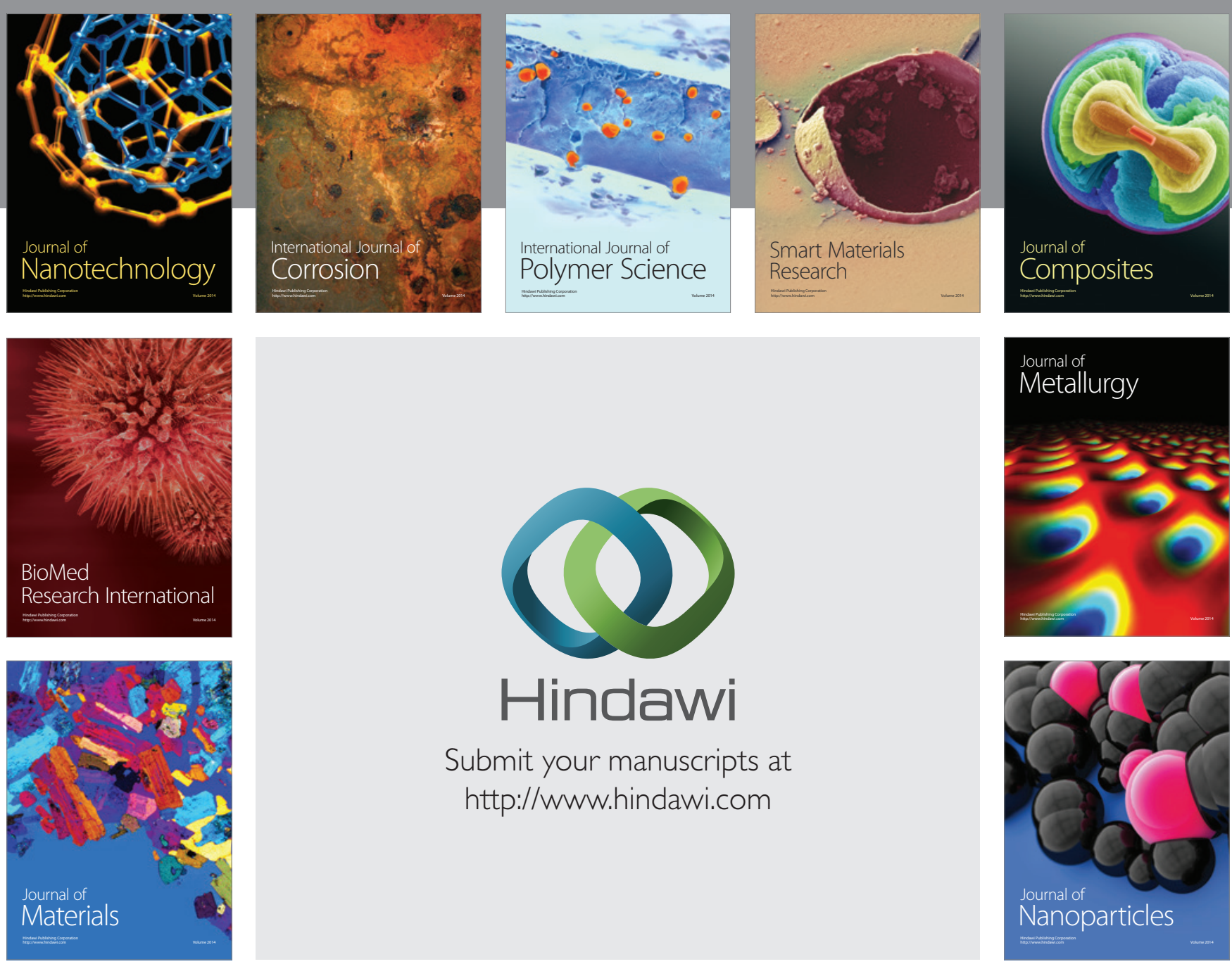

Submit your manuscripts at http://www.hindawi.com
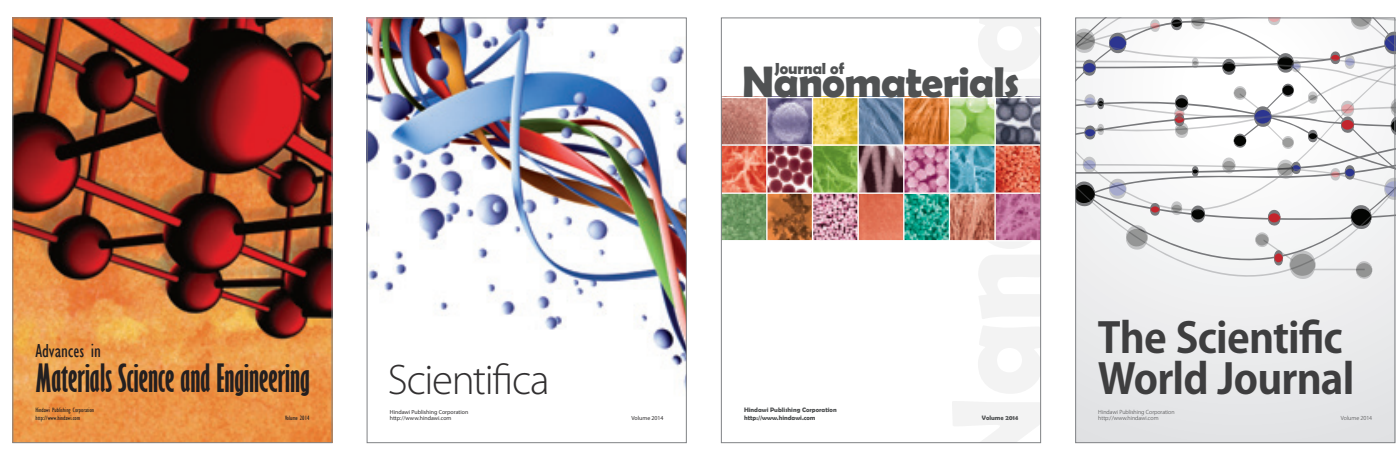

\section{The Scientific World Journal}
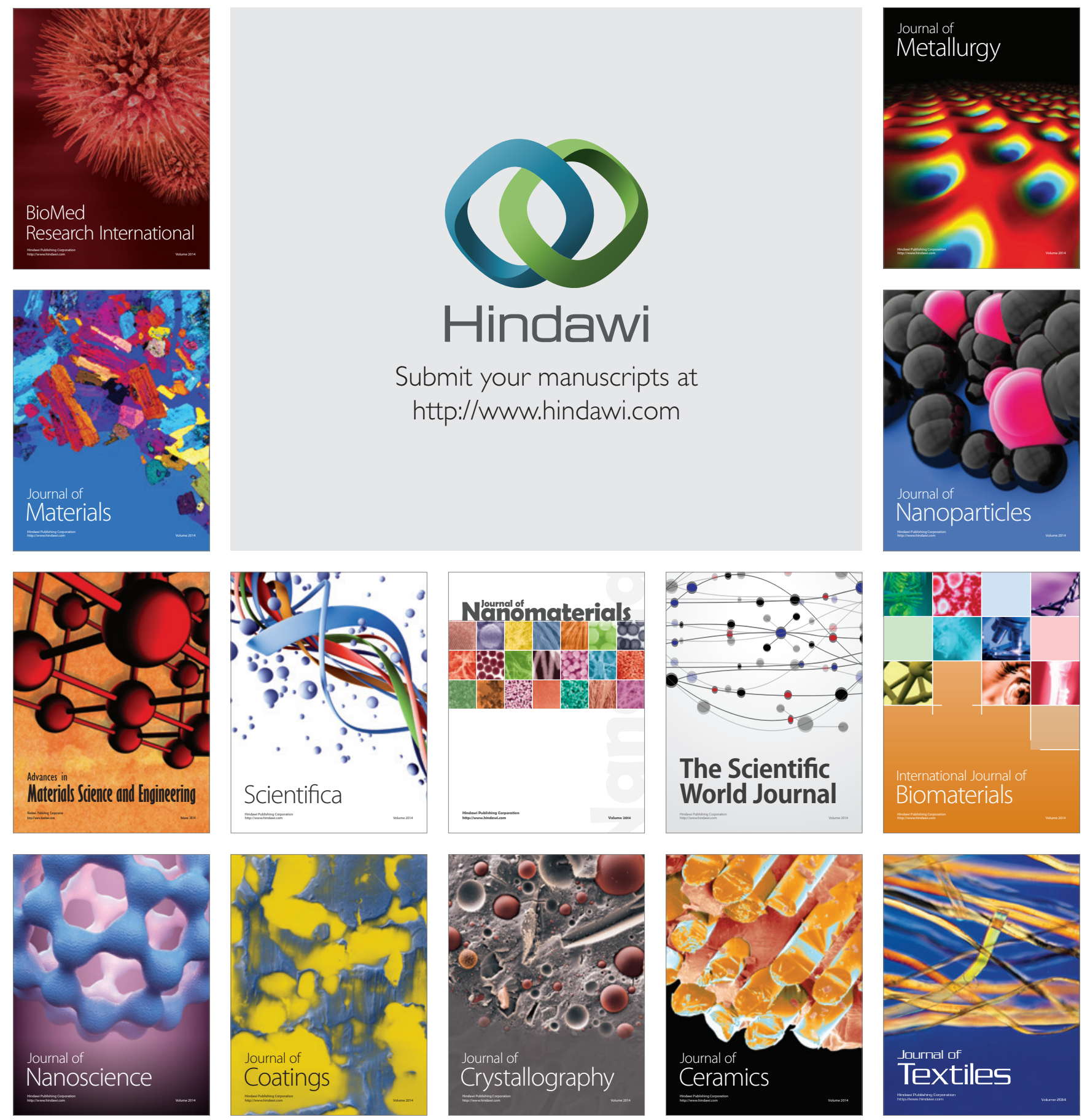